\title{
Testicular swelling leading to a diagnosis of adrenocortical carcinoma - co-incidence or causation?
}

\author{
Maria Batool1, David Fennell1, David Slattery'1, Eamon Leen², Liam Cormican², \\ Seamus Sreenan ${ }^{1}$ and John H McDermott ${ }^{1}$
}

${ }^{1}$ Academic Department of Endocrinology, Royal College of Surgeons of Ireland and 2Departments of Radiology, Respiratory Medicine, and Histopathology, Connolly Hospital, Blanchardstown, Dublin, Ireland
Correspondence

should be addressed

to M Batool

Email

mariabatool25@hotmail.com

\section{Summary}

Adrenocortical carcinoma (ACC) is a rare malignancy with an incidence of $0.7-2.0$ cases/million/year. A majority of patients present with steroid hormone excess or abdominal mass effects, and in 15\% of patients ACC is discovered incidentally. We present a case of 30-year-old otherwise asymptomatic Caucasian male who presented with a testicular swelling. Subsequent imaging and investigations revealed disseminated sarcoidosis and an $11 \mathrm{~cm}$ adrenal lesion. An adrenalectomy was performed. Histological examination of the resected specimen confirmed an ACC and also demonstrated a thin rim of adrenal tissue containing non-caseating granulomas, consistent with adrenal sarcoid.

\section{Learning points}

- This case highlights an unusual presentation of two uncommon diseases.

- This case also highlights how separate and potentially unrelated disease processes may occur concomitantly and the importance, therefore, of keeping an open mind when dealing with unusual diagnostic findings.

- We also hypothesize a potential link between the ACC and sarcoidosis in our patient.

\section{Background}

Adrenocortical carcinomas are rare adrenal malignancies, with 0.7-2.0 new cases diagnosed per one million persons per year (1). A dismal 5-year survival rate of $35 \%$ has been reported for these tumors (2). Biologically inert tumors are typically diagnosed due to the mass effect or are discovered incidentally during an abdominal imaging investigation performed for another indication (2). In cases of hormone-secreting tumors, the excess hormone secretion can determine the clinical presentation (2). Herein, we present an unusual case where testicular swelling led indirectly to a diagnosis of ACC but also to the rare diagnosis of disseminated sarcoidosis with adrenal and testicular involvement.

\section{Case presentation}

A 30-year-old otherwise asymptomatic Caucasian male presented with left testicular swelling. Background history was significant for hypertension diagnosed at the age of 18 and treated with an angiotensin receptor blocker. There was a family history of hypertension in his father, diagnosed aged 40. The patient reported losing $12 \mathrm{~kg}$ weight in the 3-4 months prior to presentation. He had no other symptoms, was a lifelong non-smoker, and had no other significant family history.

\section{Investigation}

Ultrasound of testes revealed bilateral diffuse testicular lesions (left-sided slightly bigger than right), suggestive 
of metastasis or an infiltrative process, but abdominal ultrasound also revealed an $11 \mathrm{~cm}$ complex mass superior to the left kidney, reported as being suspicious for renal cell carcinoma/abscess (Fig. 1).

An urgent CT scan of the thorax, abdomen and pelvis was arranged. This demonstrated diffuse centrilobular nodules distributed throughout both lung fields, with extensive mediastinal lymphadenopathy, and multifocal low attenuation lesions were also seen in the spleen and testes. The appearances in the lungs, mediastinum, spleen and testes were highly suggestive of sarcoidosis (Fig. 2). The CT scan also showed a complex adrenal lesion measuring $7 \times 10 \times 7 \mathrm{~cm}$.

Transbronchial biopsy was performed, and bronchial washings confirmed the diagnosis of sarcoidosis. Lung function tests showed normal spirometry, lung volumes, and gas transfer. Hormonal workup confirmed that the adrenal lesion was non-functional.

Left adrenalectomy was performed. Histology of the resected adrenal gland confirmed an ACC (Fig. 3). Weiss score was 4 (nuclear pleomorphism, capsular invasion, sinusoidal invasion and vascular invasion-each point giving a score 1), modified Weiss score of 3 (focal clear cell component, capsular invasion and abnormal mitosis). Interestingly a thin rim of uninvolved adrenal tissue remained and was found to contain non-caseating granulomas consistent with adrenal sarcoidosis (Fig. 4).

\section{Treatment}

Following a multidisciplinary discussion, the patient was commenced on mitotane therapy and responded very

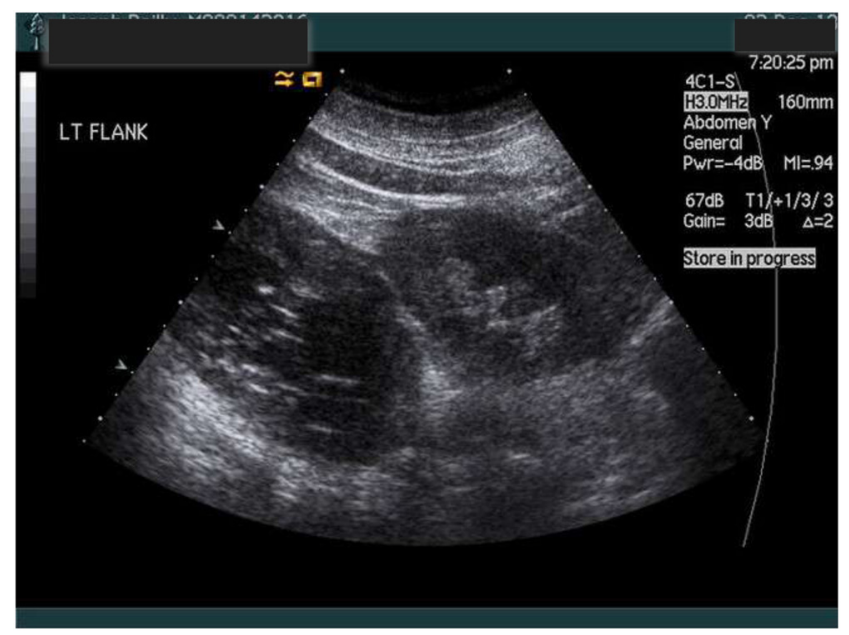

Figure 1

Ultrasound of testes showing bilateral testicular lesions but also revealing an $11 \mathrm{~cm}$ complex mass superior to left kidney.

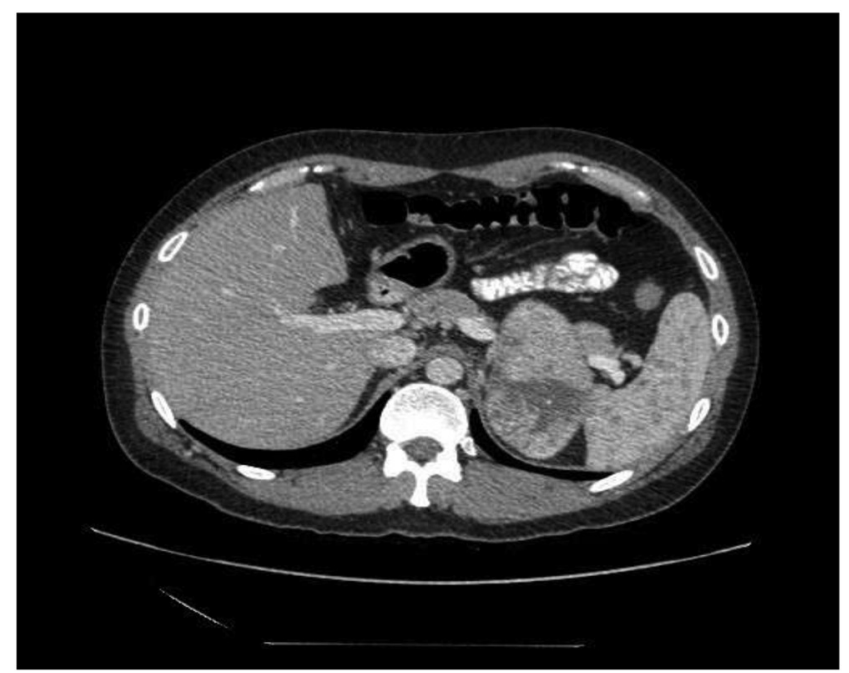

\section{Figure 2}

CT thorax, abdomen and pelvis showing left adrenal lesion, measuring $7 \times$ $10 \times 7 \mathrm{~cm}$.

well to this. He was treated with low-dose steroids for sarcoidosis, which became quiescent, with normal lung function and imaging (resolution of lymphadenopathy and testicular swelling) on follow-up.

\section{Outcome and follow-up}

Our patient is doing well with no recurrence of disease on follow-up.

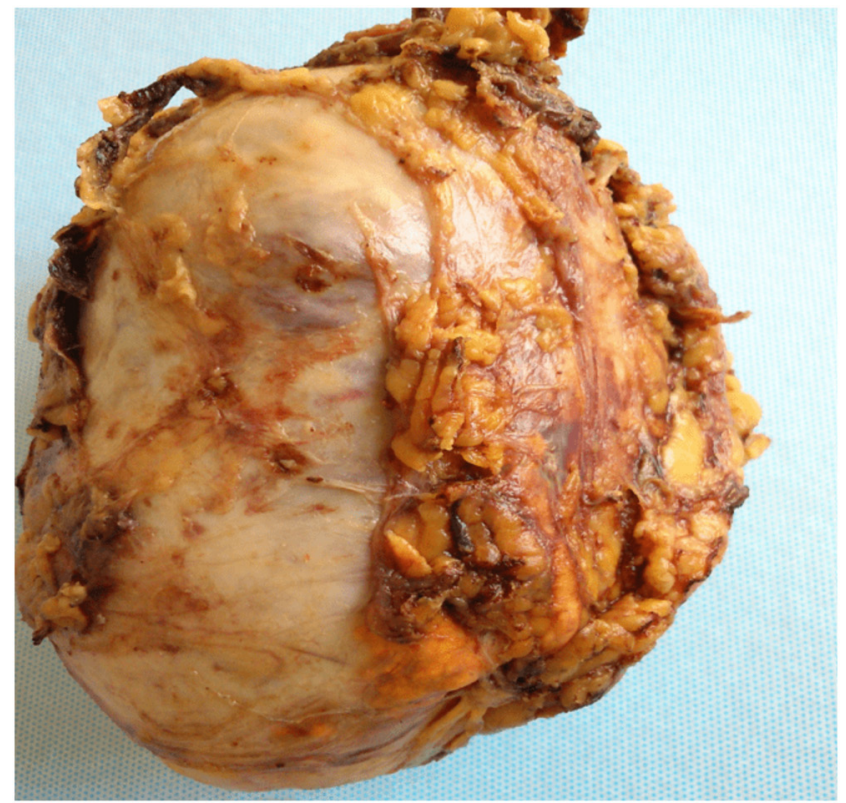

\section{Figure 3}

Gross appearance of left adrenal gland, measuring $7 \times 10 \times 7 \mathrm{~cm}$. 


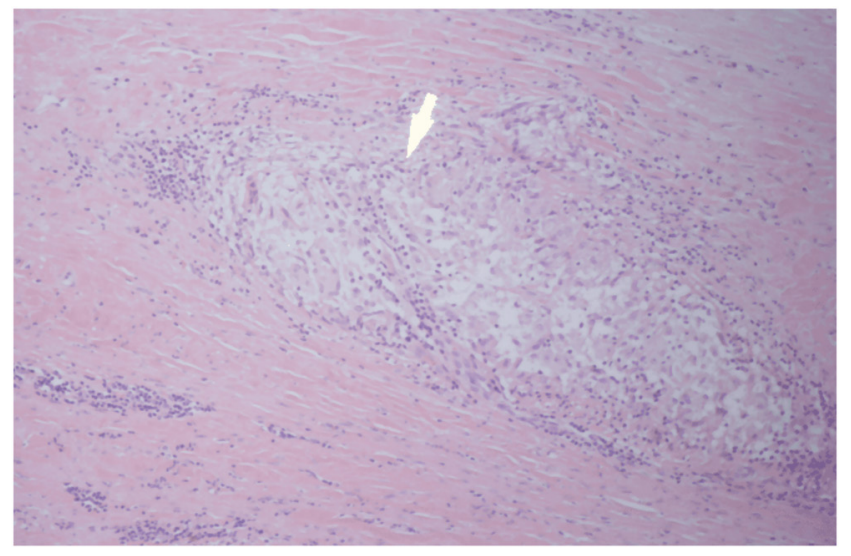

Figure 4

Histology of resected adrenal gland showing a thin rim of non-neoplastic residual tissue which contains non-caseating granuloma (a granuloma is indicated by arrow) consistent with adrenal sarcoidosis.

\section{Discussion}

Adrenal incidentalomas - defined as adrenal tumors discovered incidentally when abdominal imaging is performed for an unrelated indication - are a common clinical conundrum in modern endocrine and medical practice. The majority of adrenal incidentalomas are benign and non-functioning, but a significant proportion represents functioning and/or malignant adrenal tumors requiring treatment.

At first glance, it appears that our patient's adrenal lesion was an incidentaloma, discovered when he was having imaging performed to investigate a possible testicular cancer. His presentation was extremely unusual, however, in the eventual synchronous diagnosis of two rare conditions - disseminated sarcoidosis with testicular and adrenal involvement, and ACC.

Sarcoidosis is a multisystem inflammatory disease that can present with a diverse array of clinical manifestations $(3,4)$. The most common extrapulmonary manifestations involve the lymphoid system, eye and skin $(4,5)$, and involvement of the genitourinary tract is a rare phenomenon. Genitourinary sarcoidosis in men is reported to occur in less than $0.2 \%$ of cases and in $5 \%$ of autopsy studies.Within the genitourinary system, sarcoid granulomas are more frequently seen within the epididymis, testis, and prostate, while rarely affecting the penis, spermatic cord, or the scrotum $(3,4,6)$.

Histologically proven adrenal sarcoidosis is another rare finding. Adrenal sarcoidosis is typically not clinically significant, as adrenal function is typically preserved, and resected adrenal specimens are typically not available for histological examination in patients with disseminated sarcoid as there is usually no indication for adrenal surgery. One case report in the literature, however, describes a 50-year-old female patient with an adrenal incidentaloma requiring resection, where subsequent histological examination revealed adrenal sarcoid (7).

William of Occam, a scholastic philosopher (12851347), postulated the following - 'pluralitas non est ponenda sine necessitate', 'plurality should not be posited without necessity'. Since antiquity generations of medical scholars have taken these lines to heart particularly when finding their way through the mazes of diagnostic medicine. Since its inception, however, this dictum has had its challenges, most prominent among those being Hickam, who countered Occam's law with the following - 'A man can have as many diseases as he damn well (or darn well) pleases'. (8) If siding with Hickam, in this case, we could merely consider our patient to be extremely fortunate: a potentially fatal ACC was diagnosed earlier than would otherwise have been the case, with a huge potential impact on his long-term outcome, as an indirect result of an unusual presentation of another rare condition (genitourinary sarcoid). A review of the literature provides food for thought, however, and suggests that 'Occam's razor' should not be immediately dismissed in this case. Noreña-Rengifo et al. (9) report the case of a patient with Cushing's disease, who, following trans-sphenoidal surgery and cure of hypercortisolism, developed disseminated sarcoidosis. The authors speculate that the drop in serum cortisol levels that occurred following surgery unmasked previously quiescent sarcoidosis, which had been effectively 'auto-treated' by the excess cortisol levels present during the period of active Cushing's disease. De-differentiation resulting in loss of function has been described in other malignancies - for instance, de-differentiation of thyroid cancer can result in loss of responsiveness to radioactive iodine due to loss of thyroid differentiation features such as iodine uptake and organification. Aashiq et al. (10) have reported this phenomenon. We speculate that a similar process may have occurred in our patient. If in the earlier stages of his disease his adrenal lesion was hypersecreting cortisol, as it enlarged over time, it could have de-differentiated, with a resulting drop in serum cortisol levels; this, as was demonstrated clearly in the case referenced above, would have the potential to unmask quiescent sarcoidosis; this in turn leading to the patient's testicular swelling which ultimately led to early diagnosis and management 
of disease process. This clearly remains a matter of conjecture, however. Although our patient had a history of hypertension which developed at a young age, there was a clear family history of same, and the hypertension had developed many years prior to presentation; the only other clinical feature which could support our speculation was his loss of $12 \mathrm{~kg}$ in weight in the months leading to presentation. This, clearly, could have alternative explanations other than the spontaneous resolution of hypercortisolemia.

In summary, we present the case of a patient who presented with testicular swelling and was found to have two synchronous and rare conditions: disseminated sarcoidosis with adrenal and testicular involvement, and ACC.

\section{Declaration of interest}

The authors declare that there is no conflict of interest that could be perceived as prejudicing the impartiality of the research reported

\section{Funding}

This research did not receive any specific grant from any funding agency in the public, commercial or not-for-profit sector.

\section{Patient consent}

Written informed consent for publication of their clinical details and clinical images was obtained from the patient.

\section{References}

1 Libe R, Val P \& Ulloa-Aguirre A. Adrenocortical carcinoma (ACC): diagnosis, prognosis, and treatment. Frontiers in Cell and Developmental Biology 20153 45. (https://doi.org/10.3389/fcell.2015.00045)

2 Symeonidis D, Chatzinikolaou I, Koukoulis G, Mamaloudis I \& Tepetes K. Adrenocortical carcinoma presenting with signs of acute abdomen. Case Reports in Surgery 20132013 132726. (https://doi. org/10.1155/2013/132726)

3 Arkema EV \& Cozier YC. Epidemiology of sarcoidosis: current findings and future directions. Therapeutic Advances in Chronic Disease 20189 227-240. (https://doi.org/10.1177/2040622318790197)

4 Statement on sarcoidosis. Joint Statement of the American Thoracic Society (ATS), the European Respiratory Society (ERS) and the World Association of Sarcoidosis and Other Granulomatous Disorders (WASOG) adopted by the ATS Board of Directors and by the ERS Executive Committee, February 1999. American Journal of Respiratory and Critical Care Medicine 1999160 736-755. (https://doi.org/10.1164/ ajrccm.160.2.ats4-99)

5 Paknejad O, Gilani MA \& Khoshchehreh M. Testicular masses in a man with a plausible sarcoidosis. Indian Journal of Urology 201127 269-271. (https://doi.org/10.4103/0970-1591.82848)

6 Rohani P, Rana RK, Barot N, Yick D \& Kamangar N. Disseminated sarcoidosis with testicular involvement: a case report and literature review. Clinical Pulmonary Medicine 201421 96-100. (https://doi. org/10.1097/CPM.0000000000000027)

7 Cha YS, Kim HJ, Lee JC, Yoo J, Hwang SS \& Cho H. Left adrenal sarcoidosis. Journal of the Korean Surgical Society 201079 306-309. (https://doi.org/10.4174/jkss.2010.79.4.306)

8 Mani N, Slevin N \& Hudson A. What three wise men have to say about diagnosis. BMJ 2011343 d7769. (https://doi.org/10.1136/bmj.d7769)

9 Noreña-Rengifo BD, Gomez-Corrales JD \& Roman-Gonzalez A. Unmasking of sarcoidosis after successful management of Cushing's syndrome. Cureus 201911 e3896. (https://doi.org/10.7759/cureus.3896)

10 Aashiq M, Silverman DA, Na'ara S, Takahashi H \& Amit M. Radioiodine-refractory thyroid cancer: molecular basis of redifferentiation therapies, management, and novel therapies. Cancers 201911 1382. (https://doi.org/10.3390/cancers11091382)

Received in final form 2 April 202

Accepted 1 June 2021 